\title{
New and Facile Synthesis of Substituted Pyrrole, Pyridine, Pyrazolo[4,3-b] Pyridine, Pyrano[3,2-c] Quinoline, Napthopyran, Naphthodipyran and Coumarin Derivatives
}

\author{
F. M. A. El-Taweel ${ }^{*}$, A. A. Elagamey \\ Department of Chemistry, Facultyof Science, Damietta University, Damietta, Egypt \\ Email: *fathyeltaweel@yahoo.com \\ Received February 16, 2013; revised March 8, 2013; accepted March 12, 2013
}

\begin{abstract}
Several new pyrazolo[4,3-b] pyridines $\mathbf{7 a}$, $\mathbf{b}$ were prepared by reacting arylidenemalononitriles $\mathbf{1 a}, \mathbf{c}$ or $\mathbf{1 i}, \mathbf{j}$ with 4-nitrosoantipyrine 4. Reacting 1a, $\mathbf{b}, \mathbf{d}$ with 4-azidomethylcarbonylantipyrine 8 give 2-aminopyrrole 14. Pyrano[3,2-c] quinolines 20a, b and 23 were obtained by reacting 4-hydroxyquinoline 15 with $1 \mathbf{g}, \mathbf{h}, \mathbf{2 b}$ respectively. Reaction of $\mathbf{1}$ with naphthalenediols 24, 27 and 29 yield naphthodipyrans 26a, b, 28a, b and 30a, b respectively. Spironaphthodipyrans 32, 33 and spironaphthopyrans 36, 37 were prepared through reaction of 2a with naphthalenediols 24, 27, 34 and 35 respectively. Condensation of thioxothiazole 38 with 3,5-dibromo-2-hydroxybenzaldehyde give $2 \mathrm{H}$ chromene-3-carboxamide $\mathbf{4 2}$. 38 also reacted with 1c and 2a to give 3,5-dicyanopyridines $\mathbf{4 5}$ and $\mathbf{4 7}$ respectively. Reaction of 2-cyano- $N$ '-(1-thiophen-2-yl)ethylidene) acetohydrazide $\mathbf{4 9}$ with 1a, $\mathbf{b}$ afforded 3,5-dicyanopyridines $\mathbf{5 3}$.
\end{abstract}

Keywords: Pyrrole; Pyridine; Pyrazolo[4,3-b] Pyridine; Pyrano[3,2-c] Quinoline; Naphthopyran; Naphthodipyrans and Coumarin

\section{Introduction}

Polyfunctionally substituted aromatic and heteroaromatic derivatives are important class of compounds. Amomg these derivatives are biologically interesting antimicrobials [1,2], antimalarials [3], as pharmaceuticals [4], antitumor activity [5], as nonpeptide human deficiency virus (HIV) protease inhibitors [6] and antischistosomal agents [7]. Some years ago, our main interest was focused on a program aimed at developing of new synthetic approaches to Polyfunctionally substituted heterocycles $[8,9]$ utilizing simple, inexpensive and readily available starting materials. The present work has resulted in the formation of pyrrole, pyridine, pyrazolopyridine, pyranoquinoline, naphthodipyran and coumarin derivatives.

\section{Experimental}

All melting points are uncorrected and measured on Griffin \& George MBF010T (London) apparatus. Recorded yields correspond to the pure products. IR $(\mathrm{KBr})$ spectra were recorded on a Perkin Elmer SP-880 spectrophotometer and 1H-NMR spectra were measured on a

\footnotetext{
${ }^{*}$ Corresponding author.
}

Varian $500 \mathrm{MHz}$ at Minnesota (USA) University spectrometer in DMSO-d6 as solvent and TMS as an internal standard (Chemical shifts are reported in $\delta$ units $\mathrm{ppm}$ ). Microanalysis were performed on LECOCHN-932 and carried out in the Microanalytical Data Units at Cairo and Damietta Universities.

Synthesis of 6-aryl-1-methyl-3-oxo-1,2,3-trihydro-2phenyl-pyrazolo[4,3-b]pyridine-5-carbonitriles $(7 a, b)$

A solution of 1,2-dihydro-2,3-dimethyl-4-nitroso-1phenyl-5-oxopyrazole (4) $(0.01 \mathrm{~mol})$ and the arylidenes 1a, $\mathbf{c}$ or $1 \mathbf{i}, \mathbf{j}(0.01 \mathrm{~mol})$ in ethanol $(50 \mathrm{ml})$ containing a catalytic amount of piperidine $(0.1 \mathrm{ml})$ was heated under reflux for one hour, then left to cool. The solid products so formed were collected by filtration, recrystallized from the suitable solvents and then identified as $7 \mathbf{a}, \mathbf{b}$.

6-(5-Bromothiophen-2-yl)-2,3-dihydro-1-methyl3-oxo-1,2,3-trihydro-2-phenyl-1H-pyrazolo[4,3-b] pyridine-5-carbonitrile (7a)

Yellow crystals from ethanol/dimethylformamide, m.p. $240^{\circ} \mathrm{C}-242^{\circ} \mathrm{C}$, yield $85 \%$.IR $\left(v_{\max } / \mathrm{cm}^{-1}\right): 2225$ (conjugated $\mathrm{CN}), 1691(\mathrm{CO}) .{ }^{1} \mathrm{H}-\mathrm{NMR}\left(\mathrm{DMSO}^{-} \mathrm{d}_{6}\right)(\delta, \mathrm{ppm})$ : $3.35\left(\mathrm{~s}, 3 \mathrm{H}, \mathrm{N}-\mathrm{CH}_{3}\right), 6.97-7.59(\mathrm{~m}, 7 \mathrm{H}$, aromatic protons), 8.37 (s, $1 \mathrm{H}$, pyridine $\mathrm{H}-4)$. $-\mathrm{C}_{18} \mathrm{H}_{11} \mathrm{BrN}_{4} \mathrm{SO}$ (411.28) Calcd. C 52.57 H 2.70 N 13.62; Found C 52.36 H 3.04 N 
13.50 .

6-(4-Hydroxy-3-methoxyphenyl)-2,3-dihydro-1methyl-3-oxo-1,2,3-trihydro-2-phenyl-1H-pyrazolo [4,3-b] pyridin-5-carbonitrile (7b)

Red crystals from ethanol, m.p. $260^{\circ} \mathrm{C}-262^{\circ} \mathrm{C}$, yield $83 \%$.IR $\left(v_{\max } / \mathrm{cm}^{-1}\right): 3065$ (br, OH), 2224 (conjugated $\mathrm{CN}), 1661(\mathrm{CO}) .{ }^{-1} \mathrm{H}-\mathrm{NMR}\left(\mathrm{DMSO}-\mathrm{d}_{6}\right)(\delta, \mathrm{ppm}): 3.4(\mathrm{~s}$, $\left.3 \mathrm{H}, \mathrm{N}-\mathrm{CH}_{3}\right), 3.87\left(\mathrm{~s}, 3 \mathrm{H}, \mathrm{OCH}_{3}\right), 6.97-7.59(\mathrm{~m}, 8 \mathrm{H}$, aromatic protons), $8.37(\mathrm{~s}, 1 \mathrm{H}$, pyridine $\mathrm{H}-4), 9.64(\mathrm{~s}, 1 \mathrm{H}$, OH). $\mathrm{C}_{21} \mathrm{H}_{16} \mathrm{~N}_{4} \mathrm{O}_{3}$ (372.38).-Calcd. C $67.73 \mathrm{H} 4.33 \mathrm{~N}$ 15.05; Found C 67.64 H 4.4 N 15.13.

2-Amino-4-(aryl)-5-(1,2-dihydro-2,3-dimethyl-1phenyl-3-pyrazolin-5-oxo-4-yl)pyrrol-3-carbonitriles (14a-c)

A solution of 4-azidmethylcarbonyl-2,3-dimethyl-1phenyl-3-pyrazolin-5-one (8) $(0.01 \mathrm{~mol})$ and the appropriate amounts of $\mathbf{1 a}, \mathbf{b}, \mathbf{d}(0.01 \mathrm{~mol})$ in ethanol $(50 \mathrm{ml})$ was heated with piperidine $(0.1 \mathrm{ml})$ under reflux for one hour then left to cool at room temperature. The solid products, so formed, were collected by filtration, recrystallized and identified as 14a-c.

2-Amino-4-(5'-bromo-2'-thienyl)-5-(1,2-dihydro-2,3dimethyl-1-phenyl-3-pyrazolin-5-oxo-4-yl)pyrrol-3carbonitrile (14a)

Yellow crystals from ethanol/1,4-dioxane, m.p. $286^{\circ} \mathrm{C}$ - $288^{\circ} \mathrm{C}$, yield $65 \%$-IR $\left(v_{\max } / \mathrm{cm}^{-1}\right): 3470,3360\left(\mathrm{NH}_{2}\right.$, $\mathrm{NH}$ ), 2203 (conjugated CN), 1685 (CO), 1650 (CO).${ }^{1} \mathrm{H}-\mathrm{NMR}\left(\mathrm{DMSO}_{\mathrm{d}} \mathrm{d}_{6}(\delta, \mathrm{ppm}): 2.40\left(\mathrm{~s}, 3 \mathrm{H}, \mathrm{CH}_{3}\right), 3.33\right.$ (s, $\left.3 \mathrm{H}, \mathrm{N}-\mathrm{CH}_{3}\right), 7.45-7.63(\mathrm{~m}, 9 \mathrm{H}, 7 \mathrm{H}$, aromatic protons and $\left.2 \mathrm{H}, \mathrm{NH}_{2}\right), 8.5$ (s, $\left.1 \mathrm{H}, \mathrm{NH}\right) .-\mathrm{C}_{21} \mathrm{H}_{16} \mathrm{BrN}_{5} \mathrm{SO}_{2}$ (482.37) Calcd. C 52.29 H 3.34 N 14.52; Found C 52. 12 H 3.51 $\mathrm{N} 14.63$.

2-Amino-4-(4'-nitro-2'-pyrryl)-5-(1,2-dihydro-2,3dimethyl-1-phenyl-3-pyrazolin-5-0xo-4-yl)pyrrol-3carbonitrile (14b)

Brown crystals from DMF, m.p. $263^{\circ} \mathrm{C}-265^{\circ} \mathrm{C}$, yield 63\%.-IR $\left(v_{\max } / \mathrm{cm}^{-1}\right): 3452,3358,3137\left(\mathrm{NH}_{2}, \mathrm{NH}\right), 2191$ (conjugated $\mathrm{CN}$ ), $1680(\mathrm{CO}), 1660(\mathrm{CO}) .-\mathrm{C}_{24} \mathrm{H}_{21} \mathrm{~N}_{5} \mathrm{O}_{4}$ (443.45) Calcd. C 65.00 H 4.77 N 15.79; Found C 65.12 H $5.00 \mathrm{~N} 15.68$.

2-Amino-4-(3-chlorophenyl)-5-(1,2-dihydro-2,3dimethyl-1-phenyl-3-pyrazolin-5-oxo-4-yl)pyrrol-3carbonitrile (14c)

Yellow crystals from ethanol, 1,4-dioxane, m.p. $284^{\circ} \mathrm{C}$ - $286^{\circ} \mathrm{C}$, yield $65 \%$ - $\operatorname{IR}\left(v_{\max } / \mathrm{cm}^{-1}\right): 3441,3341\left(\mathrm{NH}_{2}\right.$, $\mathrm{NH}), 2203$ (conjugated $\mathrm{CN}$ ), 1685 (CO), 1620 (CO).$\mathrm{C}_{23} \mathrm{H}_{18} \mathrm{ClN}_{5} \mathrm{O}_{2}$ (431.87) Calcd. C $63.96 \mathrm{H} 4.20 \mathrm{~N}$ 16.22; Found C 63.89 H 4.31 N16.14.

Synthesis of $4 \mathrm{H}$-pyrano[3,2-c]quinolin-3carbonitriles (20a,b) and spiro-4H-pyrano[3,2-c] quinolin-3-carboxylate (23)

A solution of 3-acetyl-4-hydroxy-1-methyl-2-oxo-1Hquinoline (15) (0.01 mole) and ( $0.01 \mathrm{~mol})$ of either $\mathbf{1 g}, \mathbf{h}$ or ethyl 2-(2-oxoindolin-3-ylidene) cyanoacetate (2b) in ethanol/pyridine (1:1) $(60 \mathrm{ml})$, were refluxed for six hours. The solvent was then evaporated in vacuo and the precipitates formed were collected by filtration recrystallized and identified as 20a, $\mathbf{b}$ and $\mathbf{2 3}$ respectively.

4-(4-Methoxyphenyl)-5,6-dihydro-2-hydroxy-6methyl-5-oxo-4H-pyrano [3,2-c]quinolin-3-

carbonitrile (20a)

Colorless crystals from ethanol, m.p. $190^{\circ} \mathrm{C}-192^{\circ} \mathrm{C}$, yield 75\%.-IR $\left(v_{\max } / \mathrm{cm}^{-1}\right)$ : 3447, 3366, $3304(\mathrm{OH}), 2206$ (conjugated CN), 1699 (CO).- ${ }^{1} \mathrm{HNMR}$ : (DMSO-d $\left.\mathrm{d}_{6}\right)(\delta$, ppm): 3.10 (s, $\left.3 \mathrm{H}, \mathrm{CH}_{3}\right), 3.83$ (s, $\left.3 \mathrm{H}, \mathrm{CH}_{3}\right), 4.43(\mathrm{~s}, 1 \mathrm{H}$, pyran $\mathrm{H}-4), 6.90-8.16(\mathrm{~m}, 8 \mathrm{H}$, aromatic protons).$\mathrm{C}_{21} \mathrm{H}_{16} \mathrm{~N}_{2} \mathrm{O}_{4}$ (360.36) Calcd. C 69.99 H, 4.48 N 7.77; Found C 70.02 H 4.73 N 7.68.

4-(4-Bromophenyl)-5,6-dihydro-2-hydroxy-6methyl-5-oxo-4H-pyrano[3,2-c]quinolin-3carbonitrile (20b)

Faint yellow crystals from ethanol/dimethylformamide, m.p. $220^{\circ} \mathrm{C}-222^{\circ} \mathrm{C}$, yield $80 \%$.-IR $\left(v_{\max } / \mathrm{cm}^{-1}\right): 3451$, 3409, $3325(\mathrm{OH}), 2200$ (conjugated $\mathrm{CN}), 1682(\mathrm{CO})$.${ }^{1} \mathrm{HNMR}$ : $\left(\mathrm{DMSO}_{\mathrm{d}}\right)(\delta, \mathrm{ppm}): 3.0\left(\mathrm{~s}, 3 \mathrm{H}, \mathrm{CH}_{3}\right), 4.50$ (s, $1 \mathrm{H}$, pyran $\mathrm{H}-4), 7.35-8.16$ (m, $8 \mathrm{H}$, aromatic protons).$\mathrm{C}_{20} \mathrm{H}_{13} \mathrm{BrN}_{2} \mathrm{O}_{3}$ (409.23) Calcd. C 58.70 H 3.20 N 6.85; Found C 58.65 H 3.34 N 6.80.

Ethyl 2-amino-5,6-dihydro-5-oxo-6-methyl-4-(1',3'dihydro-2'H-indole-2-on)spiro-4H-pyrano[3,2-c]quino lin-3-carboxylate (23)

Yellow crystals from ethanol/DMF, m.p. $270^{\circ} \mathrm{C}-$ $272^{\circ} \mathrm{C}$, yield $76 \%$.-IR $\left(v_{\max } / \mathrm{cm}^{-1}\right): 3395,3284,3196$ $\left(\mathrm{NH}_{2}, \mathrm{NH}\right), 1688(\mathrm{CO}), 1659$ (CO), 1631 (CO). ${ }^{1} \mathrm{HNMR}:\left(\mathrm{DMSO}_{6} \mathrm{~d}_{6}\right)(\delta, \mathrm{ppm}): 0.82-0.85(\mathrm{t}, \mathrm{J}=7.5 \mathrm{~Hz}$, $\left.3 \mathrm{H}, \mathrm{CH}_{3}\right), 2.87\left(\mathrm{~s}, 3 \mathrm{H}, \mathrm{CH}_{3}\right), 3.73-3.76(\mathrm{q}, \mathrm{J}=7.5 \mathrm{~Hz}$, $\left.2 \mathrm{H}, \mathrm{CH}_{2}\right), 6.69-8.14(\mathrm{~m}, 10 \mathrm{H}, 8 \mathrm{H}$, aromatic protons and $\left.2 \mathrm{H}, \mathrm{NH}_{2}\right), 10.28(\mathrm{~s}, 1 \mathrm{H}, \mathrm{NH}) .-\mathrm{C}_{23} \mathrm{H}_{19} \mathrm{~N}_{3} \mathrm{O}_{5}$ (417.41) Calcd. C 66.18 H 4.59 N 10.07; Found C 66.22 H 4.33 N 10.13 .

General procedure for preparation of (26a,b), (28a,b) and (31a,b)

A mixture of $(0.01 \mathrm{~mol})$ of each 1,5-naphthalenediol (24), 2,6-naphthalenediol (27) or 2,7-naphthalenediol (31) and $(0.02 \mathrm{~mol})$ of the arylidenemalononitriles 1 in ethanol $(50 \mathrm{ml})$ was refluxed for thirty minutes in the presence of piperidine $(0.1 \mathrm{ml})$. The obtained solid products were collected by filtration and recrystallized from the proper solvents yielding corresponds compounds 26, 28 and 31 respectively.

2,8-Diamino-4,10-di(4-hydroxyphenyl)-4,10dihydronaphtho[1,2-b:5,6-b']dipyran-3,10dicarbonitrile (26a)

Yellow crystals from ethanol/DMF, m.p., $>300^{\circ} \mathrm{C}$, yield $60 \%$.IR $\left(v_{\max } / \mathrm{cm}^{-1}\right): 3421,3321\left(\mathrm{NH}_{2}, \mathrm{OH}\right), 2187$ (conjugated CN), $1653\left(\delta \mathrm{NH}_{2}\right) \cdot{ }^{1} \mathrm{H}-\mathrm{NMR}\left(\mathrm{DMSO}-\mathrm{d}_{6}\right)(\delta$, ppm): 4.77 (s, two equivalent pyran 4-H), $6.69-7.88$ (m, $16 \mathrm{H}, 12 \mathrm{H}$, aromatic protons and $\left.4 \mathrm{H}, 2 \mathrm{NH}_{2}\right), 9.32(\mathrm{~s}, 2 \mathrm{H}$, 
2OH). $-\mathrm{C}_{30} \mathrm{H}_{20} \mathrm{~N}_{4} \mathrm{O}_{4}$ (500.50) Calcd. C $71.99 \mathrm{H} 4.03 \mathrm{~N}$ 11.19; Found C 71.03 H 4.34 N 11.22.

2,8-Diamino-4,10-di(3-chlorophenyl)-4,10-dihydron aphtho[1,2-b:5,6-b'] dipyran-3,10-dicarbonitrile (26b)

Yellow crystals from DMF, m.p., $>300^{\circ} \mathrm{C}$, yield $63 \%$.IR $\left(v_{\max } / \mathrm{cm}^{-1}\right): 3499,3325,3197\left(\mathrm{NH}_{2}\right), 2196$ (conjugated $\mathrm{CN}), 1665\left(\delta \mathrm{NH}_{2}\right)$. $-\mathrm{C}_{30} \mathrm{H}_{18} \mathrm{Cl}_{2} \mathrm{~N}_{4} \mathrm{O}_{2}$ (537.39) Calcd. C 67.05 H 3.38 N 10.43; Found C 67.23 H 3.42 N 10.33.

3,9-Diamino-1,7-di(3-chlorophenyl)-

1,7dihydronaphtho[2,1-b:6,5-b'] dipyran-3,9-

dicarbonitrile (28a)

Yellow crystals from DMF m.p. $>300^{\circ} \mathrm{C}$, yield $64 \%$.IR: $\left(v_{\max } / \mathrm{cm}^{-1}\right): 3458,3326,3199\left(\mathrm{NH}_{2}\right), 2193$ (conjugated $\mathrm{CN}), 1657\left(\delta \mathrm{NH}_{2}\right) \cdot-\mathrm{C}_{30} \mathrm{H}_{18} \mathrm{Cl}_{2} \mathrm{~N}_{4} \mathrm{O}_{2}$ (537.39) Calcd. C 67.05 H 3.38 N 10.43; Found C 67.00 H 3.27 N 10.51.

3,9-Diamino-1,7-di(4-bromophenyl)1,7dihydronaphtho[2,1-b:6,5-b'] dipyran-3,9dicarbonitrile (28b)

Faint yellow from ethanol/DMF m.p. $>300^{\circ} \mathrm{C}$, yield 65\%.-IR $\left(v_{\max } / \mathrm{cm}^{-1}\right): 3457,33327,3197\left(\mathrm{NH}_{2}\right), 2191$ (conjugated CN). ${ }^{1} \mathrm{H}-\mathrm{NMR}\left(\mathrm{DMSO}_{-} \mathrm{d}_{6}\right)(\delta, \mathrm{ppm}): 5.34$ (s, two equivalent pyran $4-\mathrm{H}), 6.99-7.89(\mathrm{~m}, 16 \mathrm{H}, 12 \mathrm{H}$, aromatic protons and $\left.4 \mathrm{H}, 2 \mathrm{NH}_{2}\right) \cdot \mathrm{C}_{30} \mathrm{H}_{18} \mathrm{Br}_{2} \mathrm{~N}_{4} \mathrm{O}_{2}(626.29)$ Calcd. C 57.53 H 2.90 N 8.95; Found C 57.64 H 3.02 N 8.83 .

3-Amino-9-hydroxy-1-(4-hydroxyphenyl)-1Hbenzo[f]chromen-2-carbonitrile (31a)

Yellow crystals from ethanol/dimethylformamide, m.p. $260^{\circ} \mathrm{C}-262^{\circ} \mathrm{C}$, yield $75 \%$.IR $\left(v_{\max } / \mathrm{cm}^{-1}\right): 3482,3377$, $3162\left(\mathrm{NH}_{2}, \mathrm{OH}\right), 2183$ (conjugated $\left.\mathrm{CN}\right), 1651\left(\delta, \mathrm{NH}_{2}\right)$.${ }^{1} \mathrm{H}-\mathrm{NMR}$ (DMSO-d $\left.{ }_{6}\right)(\delta, \mathrm{ppm}): 4.89(\mathrm{~s}, 1 \mathrm{H}$, pyran $4-\mathrm{H})$, $6.65-7.77\left(\mathrm{~m}, 11 \mathrm{H}, 9 \mathrm{H}\right.$ aromatic protons and $\left.2 \mathrm{H}, \mathrm{NH}_{2}\right)$, 9.26, $9.8(2 \mathrm{~s}, 2 \mathrm{H}, 2 \mathrm{OH}) \cdot-\mathrm{C}_{20} \mathrm{H}_{14} \mathrm{~N}_{2} \mathrm{O}_{3}$ (330.33) Calcd. C 72.72 H 4.27 N8.48; Found C 72.82 H 4.15 N 8.51.

3-Amino-9-hydroxy-1-(4-bromophenyl)-1H-benzo [f]chromen-2-carbonitrile (31b)

Yellow crystals from DMF, m.p. $268^{\circ} \mathrm{C}-270^{\circ} \mathrm{C}$, yield $70 \%$. IR $\left(v_{\max } \mathrm{cm}^{-1}\right)$ : $3480-3338\left(\mathrm{NH}_{2}, \mathrm{OH}\right), 2189$ (conjugated $\mathrm{CN}), 1648(\delta \mathrm{NH} 2) .-\mathrm{C}_{20} \mathrm{H}_{13} \mathrm{BrN}_{2} \mathrm{O}_{2}$ (393.23) Calcd. C 61.09 H 3.33 N 7.12; Found C 61.32 H 3.51 N 7.30.

Synthesis of 2,8-Diamino-4,10-di[1',3'-dihydro-2' $H$ indol-2-on)spiro] naphtho [1,2-b:5,6-b']dipyran-3,9dicarbonitrile (32) and 3,9-Diamino-1,7-di[1',3'dihydro-2' $H$-indol-2-on) spiro]naphtho [2,1-b:6,5-b'] dipyran-2,8-dicabonitrile (33): Genaral procedure:

A suspension of $(0.01 \mathrm{~mol})$ of each of 1,5-naphthalenediol (24) or 2,6-naphthalenediol (27) and 2-(2oxoindolin-3-ylidene) malononitrile (2a) $(0.02 \mathrm{~mol})$ in ethanol $(50 \mathrm{ml})$ containing few drops of piperidine was refluxed for one hour. The solids deposited were collected by filtration, recrystallized and identified as $\mathbf{3 2}$ and 33 respectively.

2,8-Diamino-4,10-di[1',3'-dihydro-2' $H$-indol-2-on) spiro]naphtho [1,2-b:5,6-b']dipyran-3,9-dicarbonitrile
(32)

Yellow crystals from DMF, m.p. $>300^{\circ} \mathrm{C}$, yield $80 \%$.IR $\left(v_{\max } / \mathrm{cm}^{-1}\right): 3448,3368,3313\left(\mathrm{NH}_{2}, \mathrm{NH}\right), 2195$ (conjugated $\mathrm{CN}), 1713(\mathrm{CO}), 1655\left(\delta \mathrm{NH}_{2}\right) \cdot{ }^{1} \mathrm{H}-\mathrm{NMR}$ (DMSO$\left.\mathrm{d}_{6}\right)(\delta, \mathrm{ppm}): 6.73-7.94(\mathrm{~m}, 16 \mathrm{H}, 12 \mathrm{H}$, aromatic protons and $\left.4 \mathrm{H}, 2 \mathrm{NH}_{2}\right), 10.71(\mathrm{~s}, 2 \mathrm{H}, 2 \mathrm{NH}) . \mathrm{C}_{32} \mathrm{H}_{18} \mathrm{~N}_{6} \mathrm{O}_{4}(550.52)$ Calcd. C 69.81 H 3.30 N 15.27; Found C 69.76 H 3.41 N 15.30 .

3,9-Diamino-1,7-di[1',3'-dihydro-2' $H$-indol-2-on) spiro]naphtho [2, 1-b:6,5-b']dipyran-2,8-dicabonitrile (33)

Red crystals from ethanol/1,4-dioxane, m.p. $230^{\circ} \mathrm{C}-$ $232^{\circ} \mathrm{C}$, yield $85 \%$.IR $\left(v_{\max } / \mathrm{cm}^{-1}\right): 3405,3259\left(\mathrm{NH}_{2}, \mathrm{NH}\right)$, 2231 (conjugated $\mathrm{CN}), 1718(\mathrm{CO}), 1620\left(\delta \mathrm{NH}_{2}\right)$.$\mathrm{C}_{33} \mathrm{H}_{20} \mathrm{~N}_{6} \mathrm{O}_{4}$ (564.54) Calcd. C 70.21 H 3.57 N 14.89; Found C 70.10 H 3.44 N 14.78.

General method for preparation of 2-amino-4hydroxy-4-(1',3'-dihydro-2' $H$-indol-2-oxo) spironaptho [1,2-b]pyran-3-carbonitrile (36) and 2-amino-6-hdroxy-4-(1',3'-dihydro-2' $H$-indol-2-oxo) spironaphtho [1,2-b]pyran-3-carbonitrile (37):

A mixture of each of 1,4-naphthalenediol (34) or 1,6-naphthalenediol (35) $(0.01 \mathrm{~mol})$ in ethanol $(50 \mathrm{ml})$ containing piperidine $(0.1 \mathrm{ml})$ was treated with $(0.01 \mathrm{~mol})$ of 2a. The reaction mixture was refluxed for six hours and then left to cool to room temperature. The precipitates formed were collected by filtration, recrystallized and identified as naphthopyrans $\mathbf{3 6}$ and $\mathbf{3 7}$ respectively.

2-Amino-4-hydroxy-4-(1',3'-dihydro-2' $H$-indol-2oxo) spiro naptho[1,2-b]pyran-3-carbonitrile (36)

Brown crystals from ethanol/DMF, m.p. $>300^{\circ} \mathrm{C}$, yield $75 \%$.-IR $\left(v_{\max } / \mathrm{cm}^{-1}\right)$ : 3423, 3301, $3200\left(\mathrm{NH}_{2}, \mathrm{NH}\right.$, $\mathrm{OH}), 2219$ (conjugated $\mathrm{CN}), 1721(\mathrm{CO}), 1646\left(\delta \mathrm{NH}_{2}\right)$.$\mathrm{C}_{21} \mathrm{H}_{13} \mathrm{~N}_{3} \mathrm{O}_{3}$ (355.34). Calcd. C 70.98 H 3.69 N 11.83; Found C 70.62 H 3.53 N 11.69.

2-Amino-6-hdroxy-4-(1',3'-dihydro-2' $H$-indol-2-oxo) spiro naphtho[1,2-b] pyran-3-carbonitrile (37)

Colorless crystals from ethanol/1,4-dioxan, m.p. > $300^{\circ} \mathrm{C}$, yield $65 \%$.IR $\left(v_{\max } / \mathrm{cm}^{-1}\right): 3482,3328,3171$ $\left(\mathrm{NH}_{2}, \mathrm{NH}, \mathrm{OH}\right), 2199$ (Conjugated $\left.\mathrm{CN}\right), 1699(\mathrm{CO})$, $1653\left(\delta \mathrm{NH}_{2}\right) \cdot{ }^{1} \mathrm{H}-\mathrm{NMR}\left(\mathrm{DMSO}-\mathrm{d}_{6}\right)(\delta, \mathrm{ppm}): 6.43-$ $6.45(\mathrm{~d}, \mathrm{~J}=9 \mathrm{~Hz}, 1 \mathrm{H}$, aromatic proton), $6.96-7.28(\mathrm{~m}$, $7 \mathrm{H}$, aromatic protons), $7.41\left(\mathrm{~s}, 2 \mathrm{H}, \mathrm{NH}_{2}\right), 8.13-8.14(\mathrm{~d}$, $\mathrm{J}=9 \mathrm{~Hz}, 1 \mathrm{H}$, aromatic proton $), 10.05(\mathrm{~s}, 1 \mathrm{H}, \mathrm{OH}), 10.65$ (s, $1 \mathrm{H}, \mathrm{NH}) . \mathrm{C}_{21} \mathrm{H}_{13} \mathrm{~N}_{3} \mathrm{O}_{3}$ (355.34) Calcd. C 70.98 H 3.69 N 11.83; Found C 70.88 H 3.76 N 11.75.

6,8-Dibromo- $N$-[4-(2,3-dimethyl-1-phenyl-3pyrazolin-5-oxo-4-yl)-2-thioxothiazol-3-yl]coumarin-3 -carboxamide (42)

A solution of $(0.01 \mathrm{~mol})$ of thiazole derivative $\mathbf{3 9}$ and (0.01 mol) of 3,5-dibromo-2-hydroxybenzaldehde (40) in ethanol $(50 \mathrm{ml})$ containing acetic acid $(1 \mathrm{ml})$ was refluxed for one hour, then left to cool. The formed precipitate was collected by filtration, recrystallized from 
ethanol to give 42 as colorless crystals, m.p. $194^{\circ} \mathrm{C}$ $196^{\circ} \mathrm{C}$, yield $85 \%$.-IR $\left(v_{\max } / \mathrm{cm}^{-1}\right): 3400,3061(\mathrm{NH})$, 1729 (CO coumarinyl), 1660 (CO antipyrinyl).-

$\mathrm{C}_{24} \mathrm{H}_{16} \mathrm{Br}_{2} \mathrm{~N}_{4} \mathrm{~S}_{2} \mathrm{O}_{4}$ (648.34) Calcd. C 44.46 H 2.49 N 8.64; Found C 44.69 H 2.53 N 8.55.

2-Cyano-2-(4'-hydroxy-3-methoxybenzylidene)- $N$ [4-(1,2-dihydro-2,3-dimethyl-1-phenyl-3-pyrazolin5-on-4-yl)-2-thioxothiazol-3-yl] acetamide (43)

A solution of thiazole derivative $39(0.01 \mathrm{~mol})$ and the appropriate amount of aromatic aldehyde $(0.01 \mathrm{~mol})$ in ethanol $(50 \mathrm{ml})$ containing $(0.1 \mathrm{ml})$ of piperidine was refluxed for three hours, then left to cool. The resulting solid obtained on standing was collected by filtration and recrystallized from ethanol to give $\mathbf{4 3}$ as colorless crystals, m.p. $258^{\circ} \mathrm{C}-260^{\circ} \mathrm{C}$, yield $60 \%$.-IR $\left(v_{\max } / \mathrm{cm}^{-1}\right): 3250$ $(\mathrm{NH}, 2214$ conjugated $\mathrm{CN}), 1634$ (CO antipyrinyl).$\mathrm{C}_{25} \mathrm{H}_{21} \mathrm{~N}_{5} \mathrm{~S}_{2} \mathrm{O}_{4}$ (519.59) Calcd. C 57.79 H 4.07 N 13.48; Found C 57.82 H 4.34 N 13.54.

6-Amino-1-[4'-(2,3-dimethyl-1-phenyl-3-prazolin-5oxo-4-yl)-2'-thioxothiazol-3'-yl]-4-aryl-3,5-dicyano-2oxo-1H-pyridine (45)

\section{Method A:}

A solution of thiazole derivative (39) $(0.01 \mathrm{~mol})$ in ethanol $(50 \mathrm{ml})$ containing $(0.1 \mathrm{ml})$ of triethylamine, was treated with $(0.01 \mathrm{~mol})$ of cinnamonitrile 1c. The reaction mixture was refluxed for three hours, then left to cool. The solid product formed was collected by filtration and recrystalized from ethanol to give colorless crystals of 45 m.p., $290^{\circ} \mathrm{C}-292^{\circ} \mathrm{C}$, yield $65 \%$.

\section{Method B:}

A suspension of the arylidene derivative $43(0.01 \mathrm{~mol})$ in ethanol $(50 \mathrm{ml})$ was treated with malononitrile $(0.01$ $\mathrm{mol})$ and dry pyridine $(20 \mathrm{ml})$. The reaction mixture was refluxed for five hours and the solvent was concentrated in vacuo and the solid formed was recrystallized and identified (m.p. and mixed m.p. as 45).-IR $\left(v_{\max } / \mathrm{cm}^{-1}\right)$ : 3323, $3198\left(\mathrm{NH}_{2}, \mathrm{NH}\right), 2215$ (conjugated $\mathrm{CN}$ ), $1636(\mathrm{CO}$ antipyrinyl).). $-\mathrm{C}_{29} \mathrm{H}_{23} \mathrm{~N}_{7} \mathrm{~S}_{2} \mathrm{O}_{4}(597.66)$ Calcd. C $58.28 \mathrm{H}$ 3.88 N 16.40; Found C 58.11 H 4.00 N 16.32.

Formation of 2-amino-1-[4'-(2,3-dimethyl-1phenyl-3-pyrazolin-5-oxo-4-yl)2'-thioxothiazol-3'-yl]-4 (1',3'-dihydro-2'H-indol-2-on) spiro-6-hydroxy-3,5dicyanopyridine (47)

To a mixture of thiazole derivative $39(0.01 \mathrm{~mol})$ and 2-(2-oxoindolin-3-ylidene)malononitrile (2a) (0.01 mol) in ethanol $(50 \mathrm{ml})$, few drops of piperidine was added. The reaction mixture was refluxed four hours, then cooled and the formed precipitate was collected by filtration and recrystallized from ethanol/DMF to give red crystals of 47 , m.p. $192^{\circ} \mathrm{C}-194^{\circ} \mathrm{C}$, yield $70 \%$.-IR $\left(v_{\max }\right)$ $\mathrm{cm}^{-1}$ ): 3250, $3063(\mathrm{NH}), 1723$ (CO), 1641 (CO antipyrinyl). $-\mathrm{C}_{28} \mathrm{H}_{20} \mathrm{~N}_{8} \mathrm{~S}_{2} \mathrm{O}_{3}(580.64)$ Calcd. C $57.92 \mathrm{H} 3.47$ N 19.30; Found C 57.81 H 3.55 N 19.11.

Preparation of 6-amino-4-aryl-3,5-dicyano-1-[1'- (2"-thienyl)ethylid-enemino] pyridine-2-(1H)-ones (53a,b)

A solution of the hydrazone derivative $49(0.01 \mathrm{~mol})$ and $(0.01 \mathrm{~mol})$ of arylidenemalononitriles $\mathbf{1 a}, \mathbf{b}$ in ethanol $(50 \mathrm{ml})$ was heated at reflux temperature for half hour. The resulting solids were collected by filtration and recrystallized from suitable solvents to yield compounds $53 a$, b.

6-Amino-4-(5'-bromo-2'-thienyl)-3,5-dicyano-1[1'-(2"-thienyl) ethylidenemino] pyridine-2-(1H)-one (53a)

Yellow crystals from ethanol/1,4-dioxan, m.p. $216^{\circ} \mathrm{C}$ $218^{\circ} \mathrm{C}$, yield $80 \%$.-IR $\left(v_{\max } / \mathrm{cm}^{-1}\right): 3451.3362\left(\mathrm{NH}_{2}, \mathrm{NH}\right)$, 2214 (conjugated $\mathrm{CN}$ ), $1655(\mathrm{CO}), 1619\left(\delta \mathrm{NH}_{2}\right)$.$\mathrm{C}_{17} \mathrm{H}_{10} \mathrm{~N}_{7} \mathrm{SO}_{3}$ (444.33) Calcd. C 45.95 H 2.27 N 15.76; Found C 45.85 H 2.36 N 15.82 .

6-Amino-4-(4'-nitro-2'-pyrryl)-3,5-dicyano-1[1'-(2"-thienyl) ethylidenemino] pyridine-2-(1H)-one (53b)

Colorless crystals from ethanol/DMF, m.p. $248^{\circ} \mathrm{C}$ $250^{\circ} \mathrm{C}$, yield $75 \%$.-IR $\left(v_{\max } / \mathrm{cm}^{-1}\right): 3447,3320,3150\left(\mathrm{NH}_{2}\right.$, $\mathrm{NH}), 2211$ (conjugated CN), 1658 (CO).- ${ }^{1} \mathrm{H}-\mathrm{NMR}$

$\left(\mathrm{DMSO}_{6}\right)(\delta, \mathrm{ppm}): 2.43\left(\mathrm{~s}, 3 \mathrm{H}, \mathrm{CH}_{3}\right), 7.09$ - $8.08(\mathrm{~m}$, $5 \mathrm{H}, 3 \mathrm{H}$, aromatic protons and $\left.2 \mathrm{H}, \mathrm{NH}_{2}\right), 8.13(\mathrm{~s}, 1 \mathrm{H}$, aromatic proton), 8.35 (s, 1H, aromatic proton), 12.92 (s, $1 \mathrm{H}, \mathrm{NH}) \cdot-\mathrm{C}_{17} \mathrm{H}_{11} \mathrm{~N}_{7} \mathrm{SO}_{3}$ (393.38) Calcd. C $51.90 \mathrm{H} 2.82$ N 24.92; Found C 51.82 H 2.57 N 24.86.

\section{Results and Discussion}

It has been found that, arylidenemalononitriles $\mathbf{1 a}, \mathbf{c}$ reacted readily with 1,2-dihydro-2,3-dimethyl-4-nitroso-1phenyl-5-oxopyrazole(4)to give 6-aryl-1-methyl-3-oxo1,2,3-trihydro-2-phenylpyrazolo[4,3-b] pyridine-5-carbonitriles (7a, b) via hydrogen cyanide and water elimination. Structures 7a, b were assigned as reaction products based on their elemental and spectral data ( $c f$. experimental). The same products were obtained by reacting ethyl arylidenecyanoacetates $\mathbf{1 i}$, $\mathbf{j}$ with $\mathbf{4}$ via elimination of one mole of carbon monoxide, ethanol and water. Compounds 7 were assumed to be formed via addition of the active methyl group in $\mathbf{4}$ to the activated double bond in $\mathbf{1}$ to give the adducts $\mathbf{5}$ which cyclized to give the intermediates $\mathbf{6}$. The later aromatized through elimination of hydrogen cyanide or ethyl formate and water. Similar sequence for the formation of similar systems has been reported before [3,7] ( $c f$. Scheme 1).

When equimolecular amounts of arylidenemalononitriles 1a, b, d and 4-azidomethylcarbonyl-1,2-dihydro-2, 3-dimethyl-1-phenyl-3-pyrazolin-5-one (8) reacted in ethanolic/piperidine, products resulting via elimination of nitrogen from phenylpyrazol-5-oxo-4-yl)-3-aryl-aziridine2,2-dicarbonitriles (10), 3-amino-4-(aryl)-6-(2,3-dihydro1,5-dimethyl-3-oxo-2-phenyl-1H-pyrazol-4-yl)-2-oxo-2H- 
pyran-3-carbonitrile (13) and 2-amino-4-(aryl)-5-(1,2dihydro-2,3-dimethyl-1-phenyl-3-pyrazolin-5-on-4-yl) pyrrol-3-carbonitriles (14) were thus considered. The aziridine structures (10) were ruled out by ${ }^{1} \mathrm{H}-\mathrm{NMR}$ spectra of the reaction products which clearly indicates the absence of methylene groups signals at $\delta \approx 4.0 \mathrm{ppm}$. The $\alpha$-pyrone structures 13 were excluded by IR spectra which clearly indicate the absence of $v \approx 1700 \mathrm{~cm}^{-1} \alpha$ pyrone carbonyl function. Thus, the pyrrole structures (14a, b) were given for the reaction products. Compounds 14 were formed via stepwise mechanism demonstrated in Scheme 2.

Also, 3-acetyl-4-hydroxy-1-methyl-2-oxo1H-quinoline (15) reacted with arylidenes $\mathbf{1 g}, \mathbf{h}$ in ethanol/pyridine (1:1) to yield 1:1adducts for which two structures 2-amino4-(aryl)-5,6-dihydro-6-methyl-5-oxo-4H-pyrano[3,2-c] quinoline-3-carboxamide (19) and 4-(aryl)-5,6-dihydro2-hydroxy-6-methyl-5-oxo-4H-pyrano[3,2-c] quinolin-3-<smiles>[X]C(C#N)=CBr</smiles>

1

$\mathrm{Ar}$<smiles>Cc1cc([N+](=O)[O-])c[nH]1</smiles>

$\mathrm{c}, \mathrm{H}$<smiles>COc1cc(C)ccc1O</smiles><smiles>N#C[Mg]</smiles><smiles>N#[W]</smiles>

e,<smiles>Cc1ccc(O)cc1</smiles>

Ar

$\mathrm{f}$,<smiles>Cc1ccc(O)cc1</smiles>

g,<smiles>COc1ccc(C)cc1</smiles>

$\mathrm{h}$,<smiles>Cc1ccc(Br)cc1</smiles>

i,<smiles>CCOC(=O)C(C)(C)CC</smiles>

j,<smiles>COc1cc(C)ccc1O</smiles>

$$
\text { , } \mathrm{CO}_{2} \mathrm{H}_{5}
$$




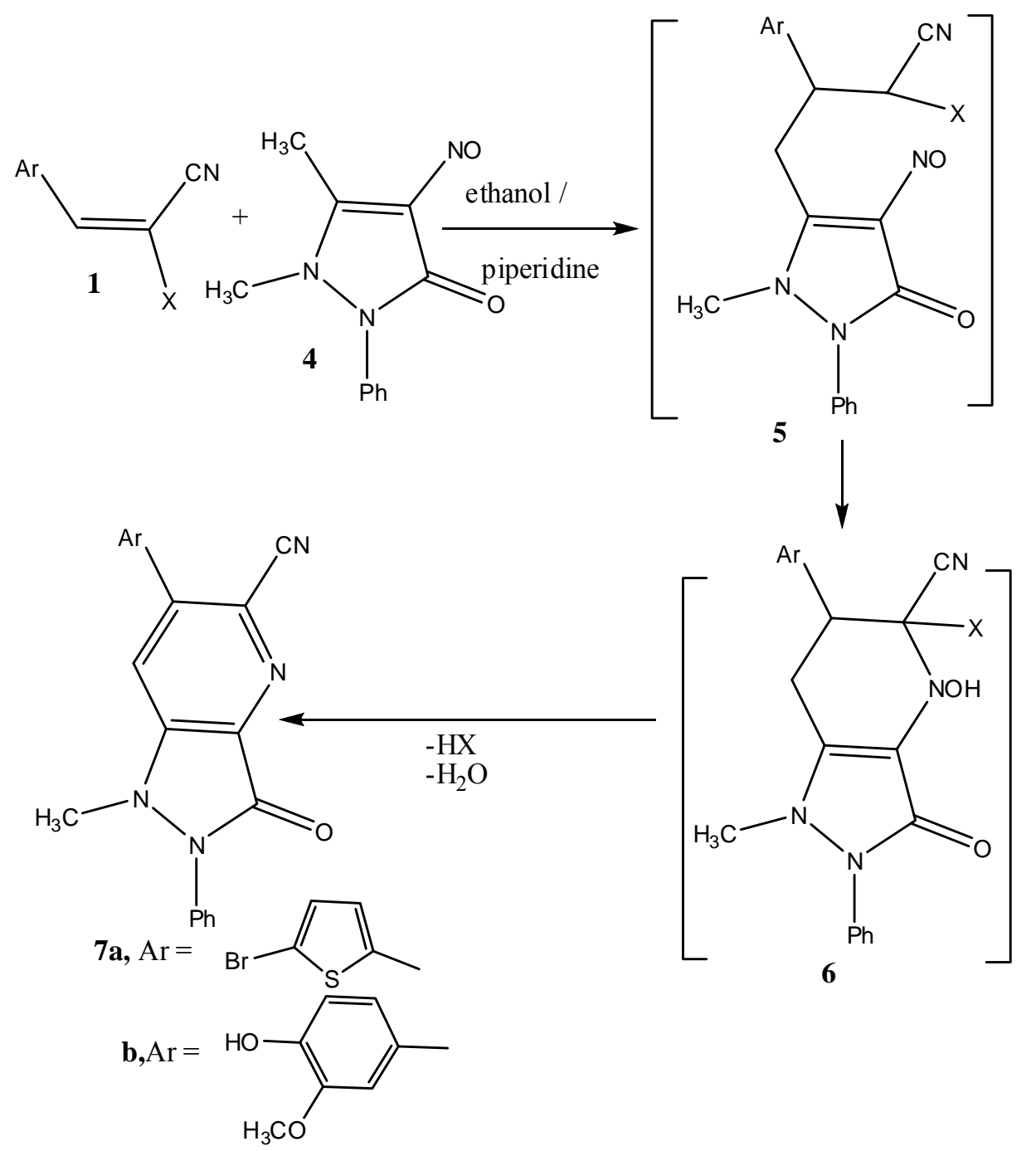

Scheme 1. Formation of pyrazolo[4,3b]pyridines $7 a, b$.

carbonitriles (20a, b) seemed possible. 4-(Aryl)-5,6-dihydro-2-hydroxy-6-methyl-5-oxo-4H-pyrano[3,2-c]quinolin-3-carbonitriles (20a, b) were established for the reaction products based on the elemental analysis and IR spectral data which showed signals at $\left(v_{\mathrm{CN}} \approx 2200-2206\right.$ $\mathrm{cm}^{-1}$ ). Structure 20 was also supported through the formation from reaction of 4-hydroxy-1-methylquinolin2(1H)one (18) with arylidenes $\mathbf{1 g}$, h utilizing the same reaction conditions. 4-(Aryl)-5,6-dihydro-2-hydroxy-6methyl-5-oxo-4H-pyrano[3,2-c] quinolin-3-carbonitriles $(20 a, b)$ were proposed to be formed via the stepwise mechanism ( $c f$. Scheme 3).

Similarly, compound 15 reacted with ethyl 2-(2oxoindolin-3-ylidene)cyanoacetate (2b) to yield ethyl 2amino-5,6-dihydro-5-oxo-6-methyl-4-(1',3'-dihydro-2' $H$ indole-2-on)spiro-4H-pyrano[3,2-c] quinolin-3-carboxylate (23).Compound 23 was authentically prepared via reaction of $\mathbf{2 b}$ with 4-hydroxy-1-methylquinolin2(1H)-one (18) using the same reaction conditions (cf.

\section{Scheme 3).}

The behavior of several napthalenediols towards arylidenemalononitrles $\mathbf{1}$ was investigated. Thus, it has been found that, arylidenemalononitriles 1 reacted readily with 1,5-naphthalenediol (24) in a molar ratio (2:1) in refluxing ethanol containing piperidine as catalyst to afford 2:1 diadducts. Two possible isomeric structures 4amino-1,5-diaryl-2,5-dihydro-8-hydroxynaphtho[1,2-b] pyrano[2',3'-b'] pyridine-3,3-dicarbonitriles (25) and 2,8diamino-4,10-di(4-hydroxyphenyl)-4,10-dihydronaphtho [1,2-b:5,6- $\left.b^{\prime}\right]$ dipyran-3,10-dicarbonitriles (26). 2,8-Diamino-4,10-diaryl-4,10-dihydronaphtho[1,2-b:5,6- $\left.b^{\prime}\right]$ dipyran-3,10-dicarbonitriles (26a, b). Structures 26a, b were established for the products based on ${ }^{1} \mathrm{H}-\mathrm{NMR}$ spectra which revealed the presence of two magnetically equivalent $4 \mathrm{H}$-pyran protons at $\delta \approx 5.0 \mathrm{ppm}$. The reaction products 25, were excluded due to two magnetically non-equivalent protons for 4H-pyran and pyridine $\mathrm{H}-2$. Similarly, arylidenemalononitriles 1 reacted with 2,6- 


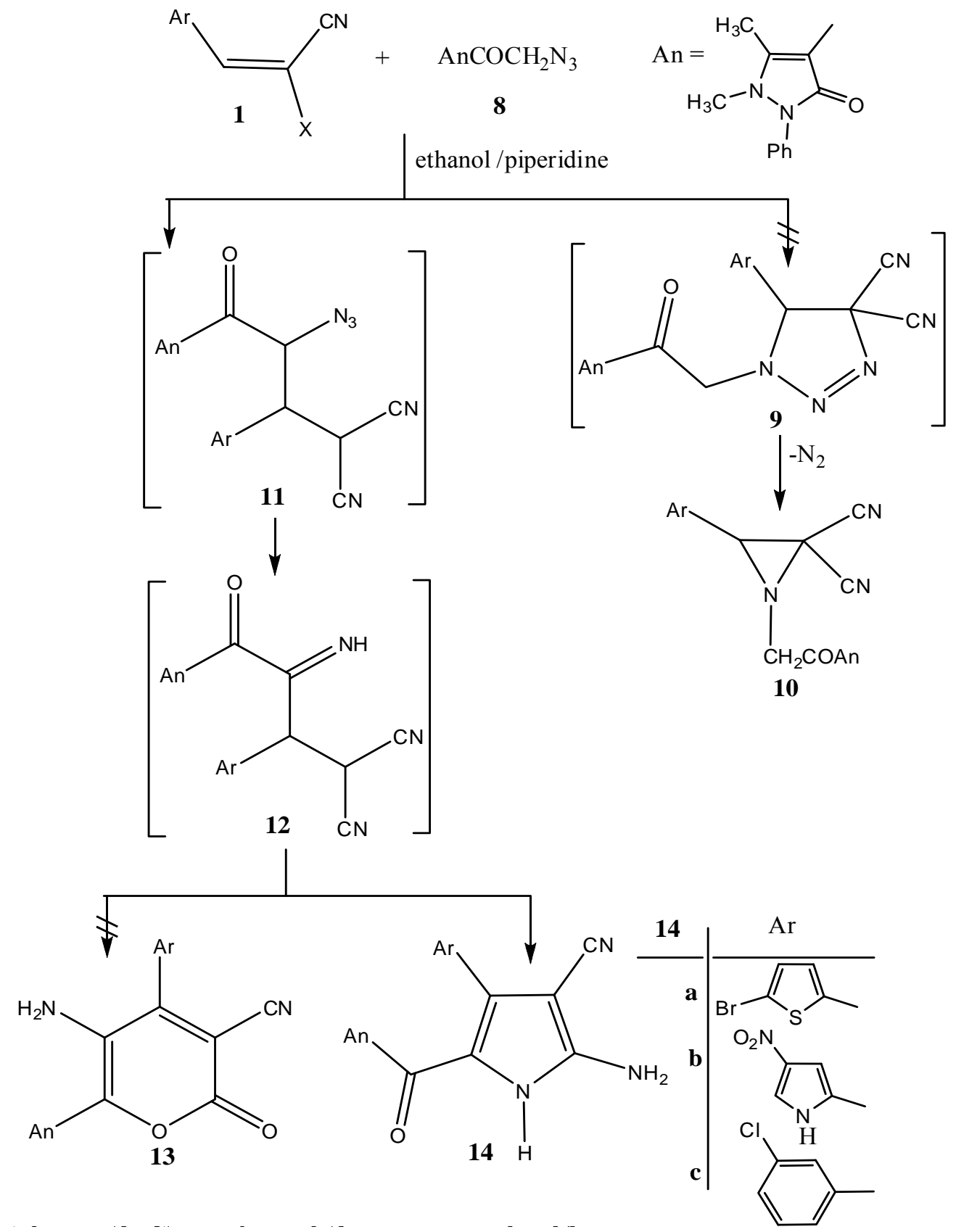

Scheme 2. Formation of 2-aminopyrroles 14a-c.

naphthalenediol (27) in ethanol catalyzed with piperidine to afford 2:1diadducts which corresponds to 3,9-diamino1,7-diaryl-1,7-dihydronaphtho[2,1-b:6,5-b'] dipyran-3,9dicarbonitriles (28). Trials to isolate a 1:1 adduct were failed. ${ }^{1} \mathrm{H}$-NMR spectra of $\mathbf{2 8}$ revealed the presence of two magnetically equivalent $4 \mathrm{H}$-pyran protons as one signal at $\delta \approx 5.34 \mathrm{ppm}$. It is of value to note that the pyran H-4 in 28 is deshielded by 0.34 ppm in comparison with 26 as a result of van der Waal's effect of the adjacent aromatic protons ( $c f$. experimental).

Also, compound 1 reacted with 2,7-naphthalenediol
(29) in ethanol and in the presence of piperidine as catalyst to afford the adducts corresponds to 3 -amino9-hydroxy-1-(aryl)-1H-benzo[f]chromen2-carbonitriles (31a, b). Structures 31a, b were preferred over possible naphthodipyrans (30) on the basis of elemental and spectral analysis. Also, structure $\mathbf{3 1}$ were found to be highly sterically hindered by the two aryl groups at C-1 and $\mathrm{C}-12$. Trials to isolate a 2:1 diadducts 30 were found unsuccessful ( $c f$. Scheme 4).

Reaction of 2-(2-oxoindolin-3-ylidene)malononitrile (2a) with 1,5-naphthalenediol (24) and 2,6-naphtha- 


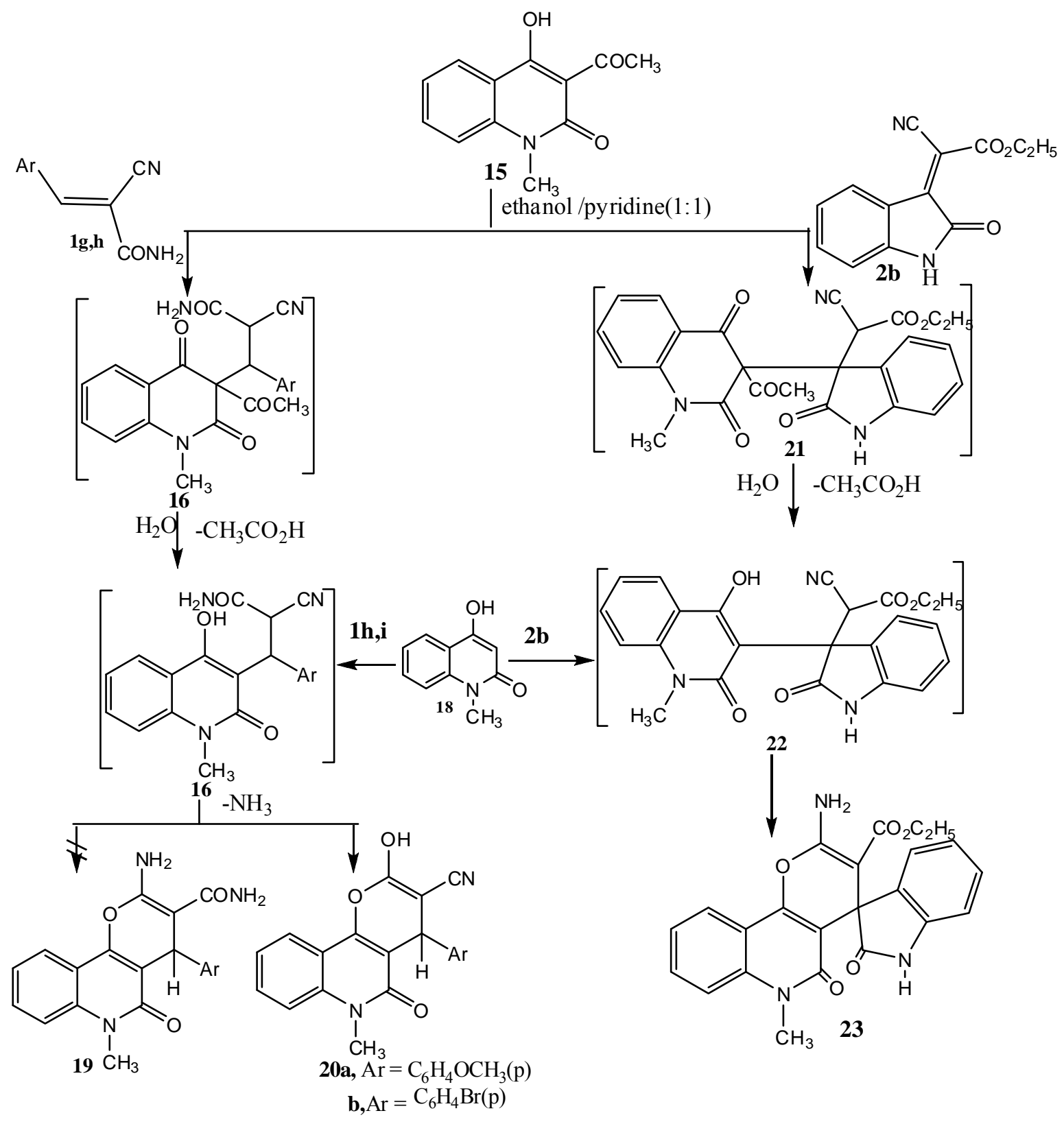

Scheme 3. Formation of pyrano[3,2-c] quinolines 20 and 23.

lenediol (27) in ethanol containing few drops of piperidine afforded a 2:1 diadducts. Structures 2,8-diamino-4,10di[1',3'-dihydro-2' $H$-indol-2-on)spiro]naphtho[1,2- $b: 5,6-$ $b^{\prime}$ ]dipyran-3,9-dicarbonitrile (32) and 3,9-dia-mino-1,7di[1',3'-dihydro-2' $H$-indol-2-on)spiro]naphtha $[2,1-b: 6,5-$ $b^{\prime}$ ]dipyran-2,8-dicabonitrile (33) ( $c f$. Scheme 5) were assigned for the reaction products based on their elemental and spectral analysis ( $c f$. experimental).

On the other hand, reaction of both of 1,4-naphthalenediol (34) and 1,6-naphthalenediol (35) with 2(2-oxoindolin-3-ylidene)malononitrile (2a) in a molar ratio $(1: 1)$ or $(1: 2)$ in ethanolic-piperidine, resulted in the formation of naphthopyans 2-amino-4-hydroxy-4-(1',3'- dihydro-2' $H$-indol-2-oxo)spiro naphtho[1,2-b]pyran-3carbonitrile (36) and 2-amino-6-hdroxy-4-(1',3'-dihydro2' $H$-indol-2-oxo)spironaphtho[1,2-b]pyran-3-carbonitrile (37) respectively ( $c f$. Scheme 5). Elemental and spectral data are compatible with naphthopyran structures 36 - 38 (cf. experimental).

Trials to prepare naphthodipyans via reacting $2 \mathbf{a}$ with 1,4-naphthalenediol (34) and 1,6-naphthalenediol (35) were found failure. This many be attributed to the molecular overcrowding arising from the difficult formation of two pyran moities located at 3,4-,5,6 and 9, 10-in positions the naphthalene ring ( $c f$. Scheme 5).

The utility of 2-cyanoethanoic acid hydrazide (3a) as 


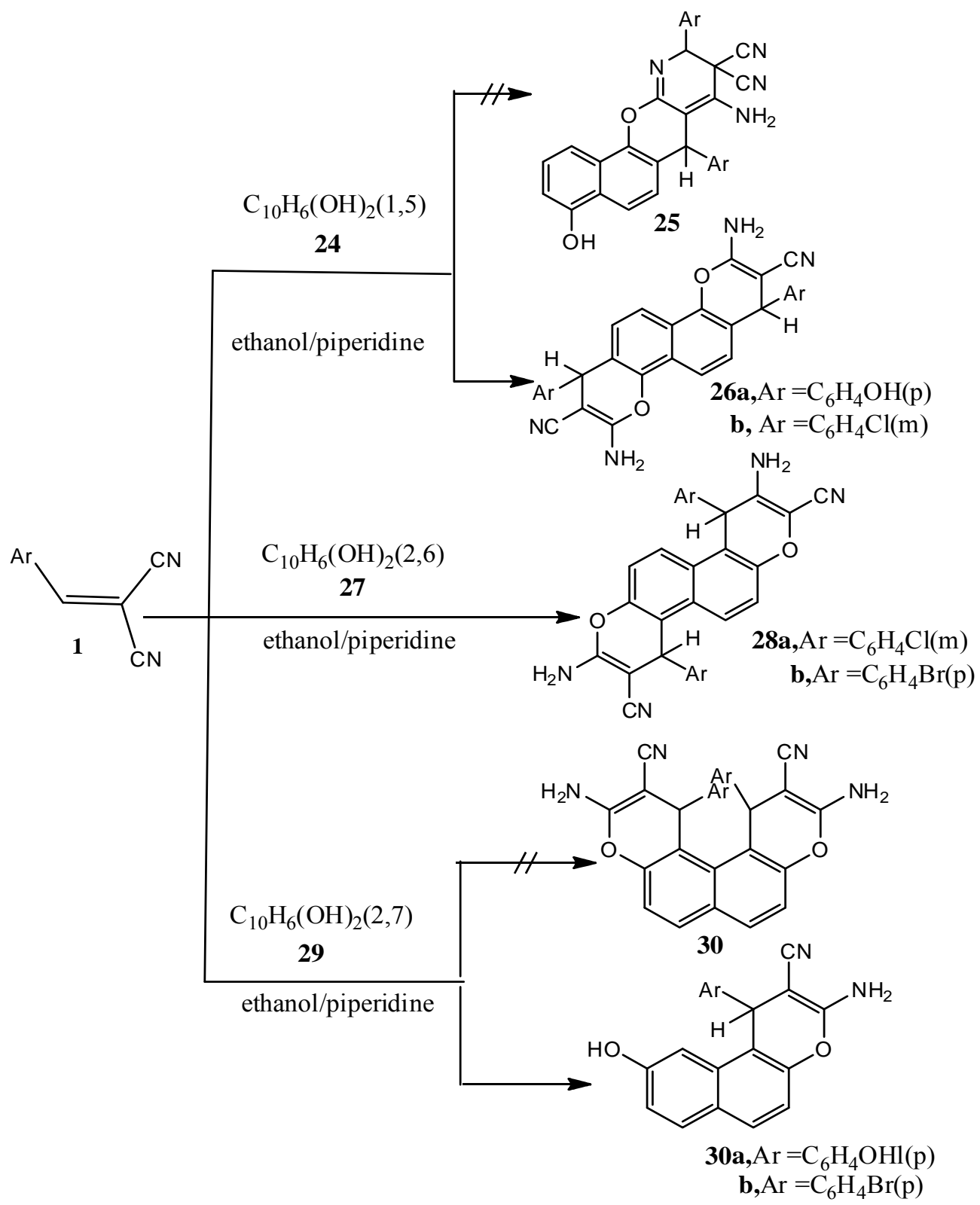

Scheme 4. Reaction of arylidenes 1 with dihydroxynaphthalenes.

starting material for synthesis of heterocyclic compounds was investigated. Thus, 2-ethanoic acid hydrazide (3a) when treated with carbon disulphide in dimethylformamide under basic conditions in potassium hydroxide/ dimethylformamide, followed by reaction with 4chloroacetyl-1-phenyl-2,3-dimethyl-3-pyrazolin-5-one (38) afforded 2-cyano- $N$-4-(2,3-dimethyl-1-phenyl-3pyrazolin-5-on-4-yl)-2-thioxothiazol-3-yl)]acetamide (39) [9].

We have studied the chemical reactivity of $\mathbf{3 9}$ towards different reagents. For example, compound 39 condensed with 3,5-dibromo-2-hydroxybenzaldehyde (40) in ethanol containing catalytic amount of acetic acid to afford 6,8-dibromo- $N$-4-[(2,3-dimethyl-1-phenyl-3-pyrazolin-5oxo-4-yl)-2-thioxothiazol-3-yl]coumarin-3-carboxamide (42). Formation of $\mathbf{4 2}$ was assumed to proceed via the formation of the intermediate arylidene derivative $\mathbf{4 1}$, followed cyclization via addition of the hydroxyl group to the cyano group and ammonia elimination.

Refluxing 2-cyano- $N$-4-(2,3-dimethyl-1-phenyl-3pyrazolin-5-on-4-yl)-2-thioxothiazol-3-yl)]acetamide (39) with the arylidenemalononitriles $\mathbf{1}$ in ethanol containing catalytic amount of triethylamine resulted in the formation of 6-amino-1-[4'-(2,3-dimethyl-1-phenyl-3prazolin-5-oxo-4-yl)-2'-thioxo-thiazol-3'-yl]-4-aryl-3,5-di cyano-2-oxopyridines (44) or the 4H-pyrans 45 . Struc- 


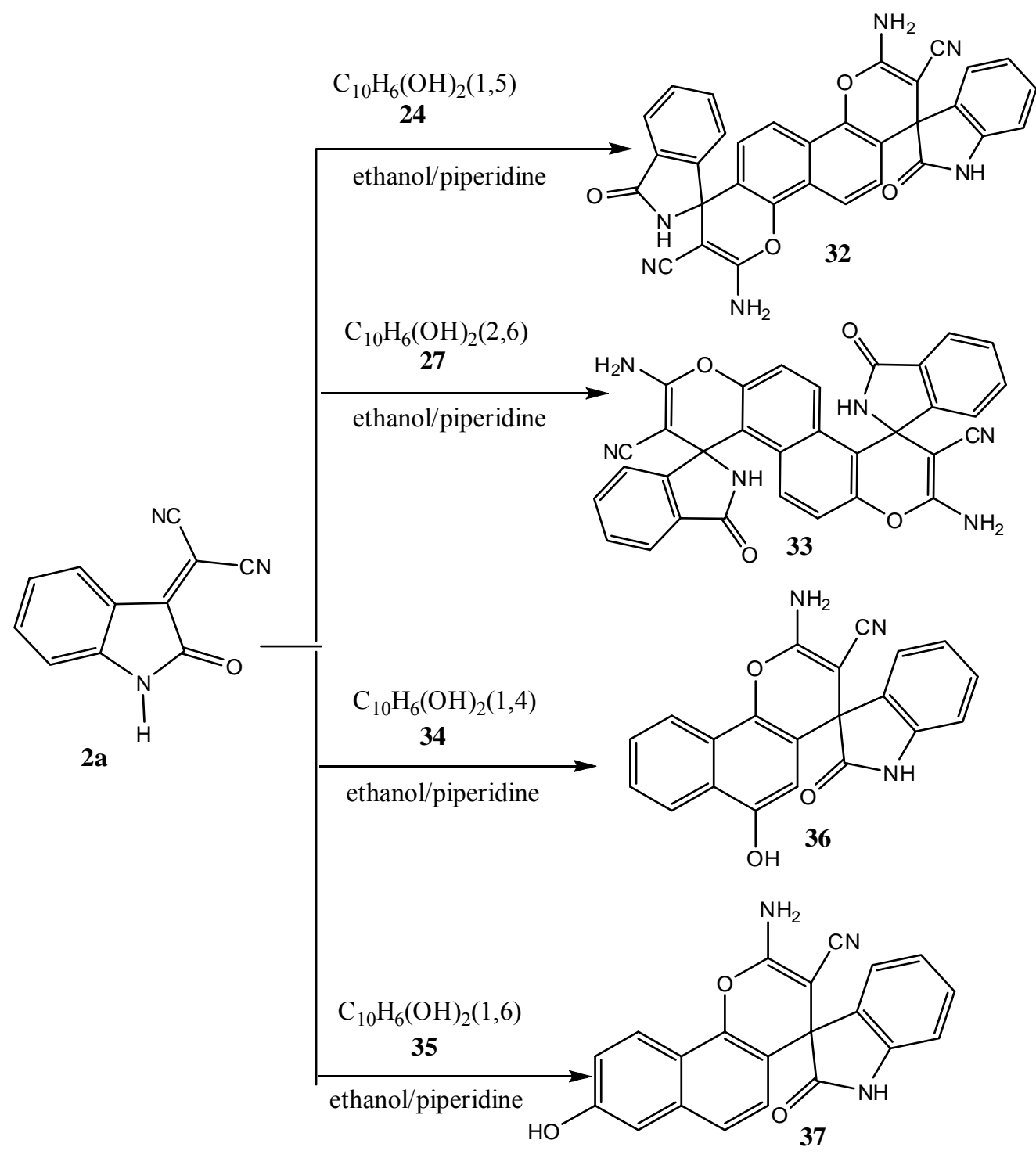

Scheme 5. Reaction of arylidenes 2a with dihydroxynaphthalenes.

tures 44 were proposed as reaction products based on their elemental and spectral analysis. If the reaction products are $45,{ }^{1} \mathrm{H}-\mathrm{NMR}$ spectra would show signal at $\approx$ 4.5 - 5.00 ppm for pyran H-4. Moreover, compounds 44 were also prepared via the reaction of the 2-cyano-2-(4'hydroxy-3-methoxybenzlidene)- $N$-[4-(1,2-dihydro-2,3-di methyl-1-phenyl-3-pyrazolin-5-oxo-4-yl)-2-thioxothiazol3-yl]acetoamide (46) with malononitrile $3 \mathbf{b}$ in ethanolicpyridine. Formation of $\mathbf{4 4}$ were suggested to take place via Michael type addition of the active methylene group in $\mathbf{3 9}$ to the $\pi$-deficient centre in $\mathbf{1}$ to give Michael adduct intermediate 43 , which cyclized and readily eliminate of one molecule of hydrogen to yield 6-amino-1-[4'-(2,3dimethyl-1-phenyl-3-prazolin-5-oxo-4-yl)-2'-thioxothiazol3'-yl]-4-aryl-3,5-dicyano-2-pyridone.

Similarly, compound $\mathbf{3 9}$ reacted with $\mathbf{2 a}$ to yield 2- amino-1-[(4'-(2,3-dimethyl-1-phenyl-3-pyrazolin-5-oxo4-yl)-2'-thioxothiazo-1,3'-yl]-4-(1',3'-dihydro-2'- $H$-indol2-on)spiro-6-hydroxy-3,5-dicyanopyridine (47) (cf. Scheme 6).

Finally, 2-cyano- $N$ '-(1-thiophen-2-yl)ethylidene)acetohydrazide (49) [10] prepared by condensing 2-cyanoethanoic acid hydrazide (3a) with 2-acetylthiophene (48), reacted with the arylidenemalonnitriles $\mathbf{1}$ in ethanol catalyzed by piperidine to give 7-(aryl)-1,2,3,5-tetrahydro-2-methyl-5-oxo-2-(thiophen-2-yl)-[1,2,4]triazolo[1, 5-a]pyridine-6,8-dicarbonitriles (51), 2-(2-(1-(thiophen2-yl)ethylidene)hydrazinyl)-6-amino-4-(aryl)-4H-pyran3,5-dicarbonitrile (52) or 6-amino-4-aryl-3,5-dicyano-1[1'-(2"-thienyl)ethylid-enemino]pyridine-2-( $1 H)$-ones (53). Structures 51 excluded by ${ }^{1} \mathrm{H}-\mathrm{NMR}$ spectrum which clearly indicate the absence of two singlets due to two 

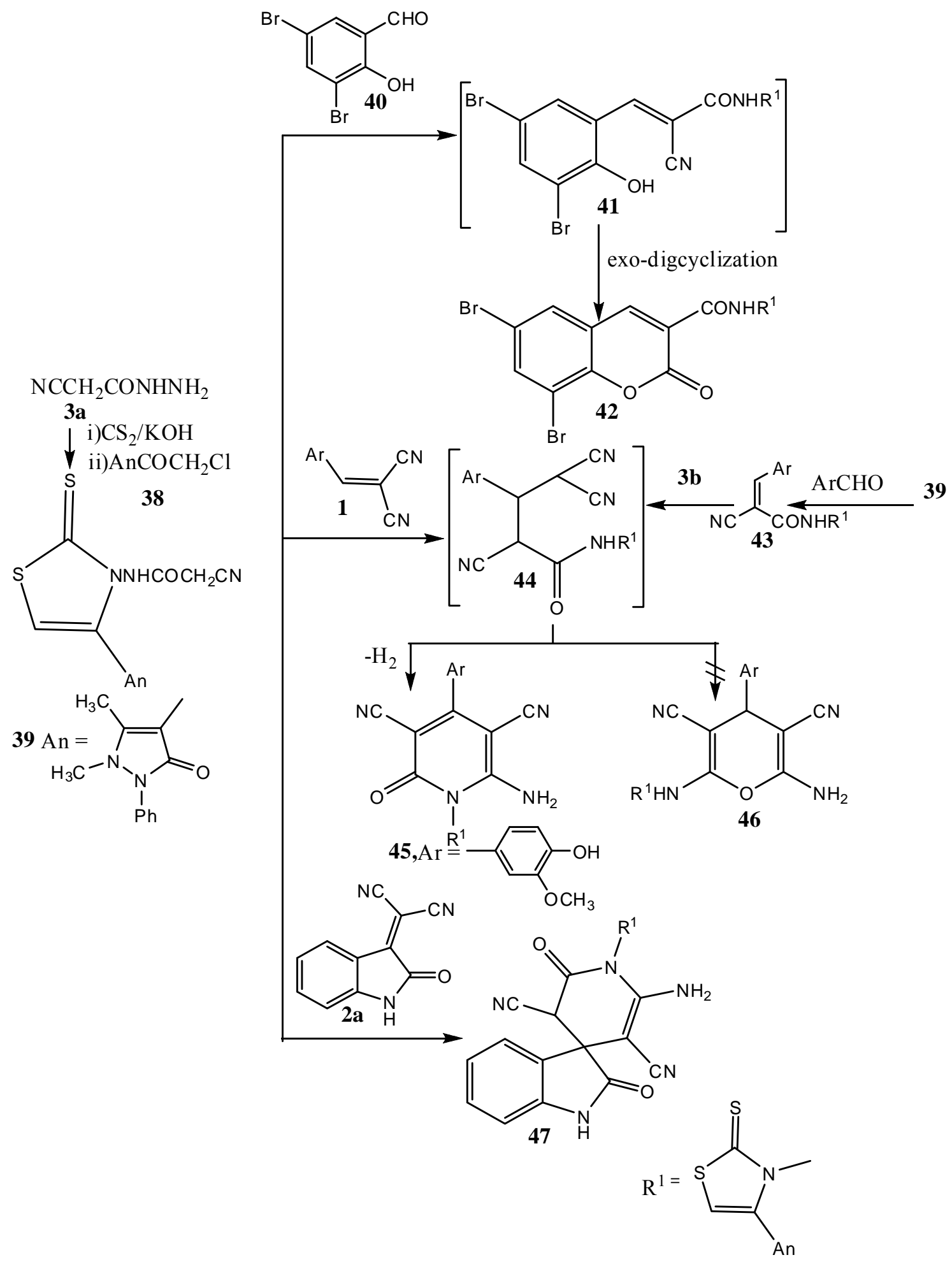

Scheme 6. Formation of coumarin 42 and pyridines 45, 47.

NH groups. Also, ${ }^{1} \mathrm{H}-\mathrm{NMR}$ spectrum of the products clearly showed the absence of 4H-pyran proton at $\delta \approx 4.5$ - $5.0 \mathrm{ppm}$ for structure 52. Consequently, the pyridine structures $\mathbf{5 3}$ were elucidated as reaction products.
Compounds 53 were suggested to be obtained via the addition of the active methylene group in $\mathbf{4 9}$ to the $\pi$-deficient carbon in $\mathbf{1}$ to give the adducts $\mathbf{5 0}$ which cyclized and dehydrogenated to give 6-amino-4-aryl-3,5- 


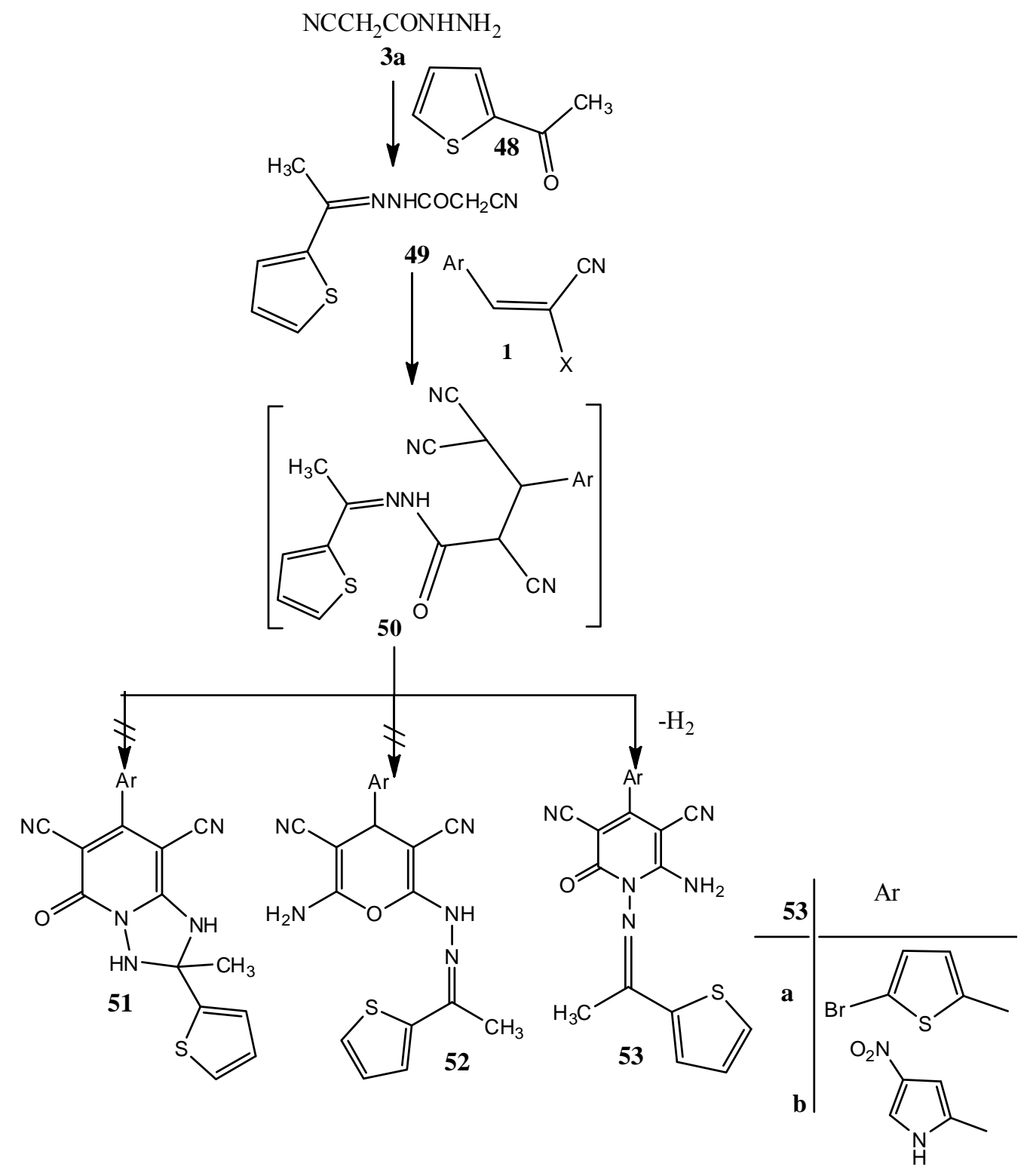

Scheme 7. Formation of pyridines 53.

dicyano-1-[1'-(2"-thienyl) ethylidenemino]-2-oxo-1Hpyridines (53) ( $c f$. Scheme 7).

\section{REFERENCES}

[1] E. Abd El-Rady and I. H. El-Azab, "Reactivity of $\beta$-Enaminoester of Benzo[f] Chromene: One Pot Synthesis of Isolated Heterocycle-Fused Derivatives of Benzo[f] Chromene," European Journal of Chemistry, Vol. 3, No. 1, 2012, pp. 81-86.

[2] H. M. Mohamed, A. H. F. Abd El-Wahab, K. A. Ahmed, A. M. El-Agrody, A. H. Bedair, F. A. Eid and M. M. Khafagy, "Synthesis, Reactions and Antimicrobial Activities of 8-Ethoxycoumarin," Molecules, Vol. 17, No. 1, 2012, pp. 971-988. doi:10.3390/molecules 17010971
[3] B. F. Abdel-Wahab, R. E. Khidre, A. A. Farahat and A. S. El-Ahl, "2-Chloroquinoline-3-aldehyde: Synthesis, Reactions and Applications," Arkivoc, Vol. 1, 2012, pp. 211276.

[4] F. M. A. El-Taweel, "Novel and Facile Synthesis of Thiophene,2H-pyran-2-one, Benzimidazo[1,2-a]pyridine and Pyridine Derivatives," Phosphorus, Sulfur and Silicon, Vol. 179, No. 1, 2011, pp. 1276-1277. doi:10.1080/10426500490468083

[5] M. M. Ghorab and M. S. Al-Said, "Synthesis and Antitumor Activity of Some Novel Hydrazide,1,2-dihydropyridine, Chromene and Benzochromene Derivatives," Journal of Heterocyclic Chemistry, Vol. 49, 2012, pp. 272-280.

[6] J. Tois, M. Vahermo and A. Koskinen, "Novel and Con- 
venient Synthesis of 4(1H)quinolones," Tetrahedron Letter, Vol. 46, No. 5, 2005, pp. 735-737.

[7] M. M. Abdelkhalik, A. M. Eltoukhy, S. M. Agamey and M. H. Elnagdi, "Enaminones as Building Blocks in Heterocyclic Synthesis of Nicotinic Acid: New Synthesis of Nicotinic Acid and Thienopyridine Derivatives," Journal of Heterocyclic Chemistry, Vol. 41, No. 3, 2004, pp. 431-435. doi:10:1002/ihet.5570410321

[8] E. S. Othman, "Some Nucleophilic Cyclization Reactions with 3-[4-Benzo[1,3]dioxolylmethylene)pyrazolyl]quinoline," Acta Chimica Slovonica, Vol. 50, 2003, pp. 15-28.
[9] F. M. A. El-Taweel, "Studies with Quinolines: New Syhthetic Routes to $4 H, 5 H, 6 H, 9 \mathrm{H}-\mathrm{Benzo}[\mathrm{ij}]$ pyrano[2,3-b] quinolizine-8-one,4H-pyrano[2,3- $b] 2 \mathrm{H}$-pyran-2-one and Pyranopyridoquinoline Derivatives," Journal of Heterocyclic Chemistry, Vol. 42, No. 5, 2005, pp. 943-946. doi:10.1002/jhet.5570420529

[10] A. A. Elagamey, A. A. El-Taweel and R. A. N. Abu El-Enein, "New Synthetic Routes to 1,3,4-Thiadiazole Derivatives," Phosphorus, Sulfur and Silicon, Vol. 181, 2006, Article ID: 21552176. 